\title{
SHOCK EXCURSION IN TRANSONIC CONVEX-CORNER FLOWS
}

\author{
KUNG-MING CHUNG \\ Aerospace Science and Technology Research Center, National Cheng Kung University \\ 2500 Section 1, Chung-Cheng South Road, Guiren district, Tainan, Taiwan \\ kmchung@mail.ncku.edu.tw \\ PO-HSIUNG CHANG \\ Temasek Laboratories, National University of Singapore \\ 5A Engineering Drive 1, \#09-02, Singapore 117411 \\ kiby716@gmail.com \\ KEH-CHIN CHANG \\ Department of Aeronautics and Astronautics, National Cheng Kung University \\ 1 University Road, east district, Tainan, Taiwan 701 \\ kcchang@mail.ncku.edu.tw
}

Published 22 June 2016

\begin{abstract}
A typical feature on a transonic airfoil is associated with a quasi-normal shock on the upper surface. When a shock is strong enough, development of a separation bubble changes the flowfield significantly. High intensity of pressure fluctuations is associated with unsteady shock wave. An experimental study was conducted to investigate transonic convex-corner flows, including singleand round-convex corners. Peak pressure fluctuations and zero-crossing frequency are associated with incoming Mach number, total turning angle, and separation length. In the cases of R100 and R200, the level of zero-crossing frequency decreases with increasing turning angle as observed in the single convex-corner flows. An increase in the level of pressure fluctuations corresponds to lower zero-cross frequency. In the cases of R300, there is a roughly constant level of surface pressure fluctuations or the variation of pressure fluctuations with the turning angle is less significant. The zero-crossing frequency would be independent of turning angle in the cases of R300.
\end{abstract}

Keywords: Convex corner; pressure fluctuations; shock excursion.

\section{Introduction}

Aircraft designs have employed flaps for takeoff and landing performance and ailerons for routine turning maneuver. Deflected control surfaces can also be employed in combination to provide variable-camber control during cruise flight ${ }^{1}$. In the previous studies $^{2-9}$, a sharp convex corner was adopted as an idealized configuration that models

This is an Open Access article published by World Scientific Publishing Company. It is distributed under the terms of the Creative Commons Attribution 3.0 (CC-BY) License. Further distribution of this work is permitted, provided the original work is properly cited. 
the deflection of an upper control surface. However, the presence of a short region of convex surface curvature would affect the flow structure, e.g., upstream expansion, downstream compression, shock-induced, boundary-layer separation and flow unsteadiness. In the present study, the surface pressure measurements were conducted to investigate the shock excursion of convex-corner flows. For the round convex-corner flows, a short region of convex surface curvature was presented and the total turning angles were the same as those of sharp convex corners. Before discussing results of the present study, brief details of the experiment are outlined next.

\section{Experimental Setup}

\subsection{Transonic wind tunnel}

Experiments were conducted in the blowdown-type transonic wind tunnel at Aerospace Science and Technology Centre, National Cheng Kung University (ASTRC/NCKU). The major components of the facility consist of air compressors, air dryers, a cooling water system, storage tanks, and a tunnel. The air storage volume of the three storage tanks is up to $180 \mathrm{~m}^{3}$ at $5.15 \mathrm{MPa}$. The dew point of high-pressure air passing through the air dryers is maintained at $-40{ }^{\circ} \mathrm{C}$ under normal operating conditions. This tunnel has a constant-area test section measuring $600 \times 600 \mathrm{~mm}$ at the cross-section and $1500 \mathrm{~mm}$ in length. The constant-area test section was enclosed within a plenum chamber was assembled with solid sidewalls and perforated top/bottom walls. The testing freestream Mach number $M$ is $0.64,0.70,0.83$ and $0.89 \pm 0.01$, and the unit Reynolds number is up to $24.1 \times 10^{6} / \mathrm{m}$. In all of the test cases, stagnation pressure $p_{o}$ and stagnation temperature $T_{o}$ are $172 \pm 0.5 \mathrm{kPa}(25 \mathrm{psi})$, and ambient temperature, respectively.

\subsection{Test models}

The test model, which was supported by a single sting mounted on the floor of the test section, was composed of a 4-deg sharp leading edge flat plate and an interchangeable instrumentation plate. The flat plate was employed to obtain a naturally developed turbulent boundary layer. To measure the surface pressure, one row of 19 pressure taps was installed in the instrumentation plate. These pressure taps of $6 \mathrm{~mm}$ apart and $2.5 \mathrm{~mm}$ diameter were drilled along the centerline of each instrumentation plate perpendicular to the test surface for flush-mounted pressure transducers. Further, to prevent crossflow from sidewall interference, two aluminum side fences were attached at both sides of the instrumentation plate. The sharp convex-corner flow with an upstream flat plate of $450 \mathrm{~mm}$ is denoted as the baseline case $\mathrm{c}^{2-4}$.

The instrumentation plate was $150 \mathrm{~mm}$ (wide) $\times 170 \mathrm{~mm}$ (long) and the virtual convex corner was located $500 \mathrm{~mm}$ from the leading edge of the flat plate. Nine round corner models were fabricated with radii $\mathrm{R}=100 \mathrm{~mm}$ (R100), $200 \mathrm{~mm}$ (R200) and 300 $\mathrm{mm}(\mathrm{R} 300)(\delta / \mathrm{R}=0.024-0.073)$. The region of convex surface curvature ranged from $x^{*}$ $= \pm 1.55$ to $x^{*}= \pm 5.55$. The convex corner angle $\eta(13-, 15-$ and 17-deg) was given from the intersection of the tangential lines (virtual corner apex), as shown in Fig. 1 


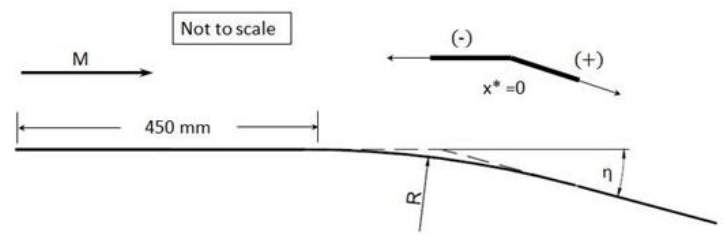

Fig. 1. Round convex-corner configuration.

\subsection{Instrumentation and Data Acquisition System}

The mean and fluctuating pressure measurements were performed using dynamic pressure transducers (Kulite model XCS-093-25A, B screen) powered by a Topward electronic system (Model 6102) with a power supply of $15.0 \mathrm{~V}$. These pressure transducers have a nominal outer-diameter of $2.36 \mathrm{~mm}$ and a pressure sensing element with a diameter of $0.97 \mathrm{~mm}$. All pressure transducers were flush mounted along the centerline of the instrumentation plate surface. The spacing between pressure transducers is $6 \mathrm{~mm}$, which is shorter than that of shock excursion (approximately $2 \delta$ ). External amplifiers (Ecreon Model E713) were employed to improve the signal-to-noise ratio. With a gain of 20, the roll-off frequency was set at approximately $140 \mathrm{kHz}$. For the data acquisition system, a NEFF 620 Instruments system and a National Instruments (NISCXI) high-speed data acquisition module were employed. The test conditions were recorded by the NEFF 620 Instruments system, while the NI-SCXI modules with LabView program were employed for surface pressure measurements. The typical sampling rate in the present study is $200 \mathrm{ksamples} / \mathrm{s}$ (or $5 \mu \mathrm{s}$ ). All input channels were triggered simultaneously by using an input channel as the trigger source. The estimated uncertainty of the experiments (based on the flat-plate case) was 2.76 and $0.46 \%$ for the static pressure coefficient $C_{p}$ and surface pressure fluctuation coefficient $\sigma_{p} / p_{w}$, respectively.

\subsection{Data Reduction (Two-Threshold Method, TTM)}

It has been well known that a typical feature of shock-induced turbulent separation is the wall-pressure signals may result from superposition or the coupling of relatively lowfrequency, high-amplitude fluctuations caused by the motion of the shock on the highfrequency, low-amplitude fluctuations of the undistributed boundary layer. To isolate the shock-motion component of the pressure signal, several studies have employed conditional sampling algorithms ${ }^{10-13}$. The typical feature is the conversion of the pressure signal into a "boxcar" of amplitude unity and varying frequency. One such algorithm, which separates pressure fluctuations caused by the shock motion from those of the boundary layer, is based on a two-threshold method (TTM) boxcar conversion technique. The shock zero-cross frequency $f_{c}$ is given by

$$
f_{c}=\frac{1}{T_{m}}=\frac{1}{(1 / N) \sum_{i=1}^{N} T_{i}}
$$


where $T_{i}$ is the $i$ th shock period, as defined in Fig. $2^{14}$, and $N$ is the number of shocks.

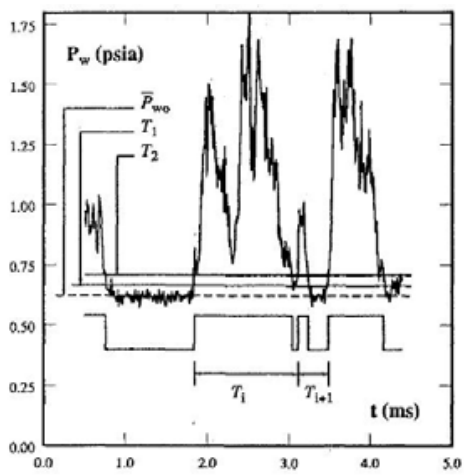

Fig. 2. Boxcar conversion technique14.

Dolling and Brusniak ${ }^{15}$ performed a sensitivity analysis using the values of $T_{1}=\bar{P}_{w o}+n \sigma_{P_{w o}}(0 \leq n \leq 3)$ and $T_{2}=\bar{P}_{w o}+m \sigma_{P_{w o}}(3 \leq m \leq 9)$, where $\bar{P}_{w o}$ is the mean pressure of the undisturbed turbulent boundary layer, and $\sigma_{P_{w o}}$ is the rms value of the turbulent boundary-layer fluctuations. Those studies also determined that setting $T_{1}=\bar{P}_{w o}+3 \sigma_{P_{w o}}$ and $T_{2}=\bar{P}_{w o}+6 \sigma_{P_{w o}}$ provided thresholds above which there was little change in $f_{c}$ (these threshold settings were used in this study). A boxcar is generated when $P_{w}(t)>T_{2}$ and it is terminated when $P_{w}(t)<T_{1}$. Thus, the boxcar assumes a value of 1 to signify a shock passage in the upstream direction, which is called the rise time. Similarly, the boxcar assumes a value of 0 to signify downstream shock passages and is called the fall time. Hence, each shock passage is characterized by a rise time and a corresponding fall time.

\section{Results and Discussion}

\subsection{Surface Pressure Fluctuations}

At $M=0.70$ at $\eta=13-\mathrm{deg}$, the distribution of normalized surface pressure fluctuations $\sigma_{p} / p_{w}$ are shown in Fig. 3. Here $x^{*}=x / \delta$ denotes the normalized streamwise distance measured along the body surface from the corner apex. It can be seen that $\sigma_{p} / p_{w}$ increases upstream of the sharp corner (baseline case) and reaches the maximum at a location immediately downstream. The level of $\sigma_{p} / p_{w}$ quickly returns to the value of the upstream undisturbed boundary layer. For the round corner cases, the location of occurrence of maximum surface pressure fluctuation is slight further downstream than the baseline case and the amplitude of the peak pressure fluctuations $\left(\sigma_{p} / p_{w}\right)_{\max }$ decreases, particularly with increasing $R$. In the upstream region $\left(-5<x^{*}<0\right)$ and the downstream region $\left(4<x^{*}<11\right)$, the amplitude of surface pressure fluctuations is independent of convex surface curvature. 


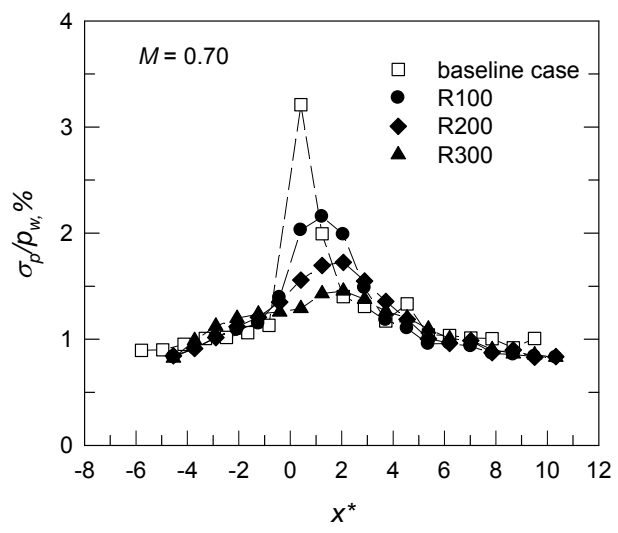

Fig. 3. Distributions of pressure fluctuations, $M=0.70, \eta=13-\mathrm{deg}$.

At $M=0.83$ and $\eta=15$-deg (separated-boundary layer), as shown in Fig. 4, peak pressure fluctuations are observed downstream of the corner in both the baseline and the round corner cases. These fluctuations are essentially related to adverse pressure gradient induced by shock wave, oscillatory motion of the separation bubble, and shock unsteadiness ${ }^{16-18}$. It is also noted that the discrete peaks implies that shock unsteadiness is a localized phenomenon with a spacing of $2 \delta$ in the streamwise direction. With an increase in $R$, the amplitude of $\left(\sigma_{p} / p_{w}\right)_{\max }$ decreases and it is observed at further downstream locations. Lower $\left(\sigma_{p} / p_{w}\right)_{\max }$ corresponds to a decrease in overall inviscid shock strength (or a lower peak Mach number) for the test cases of round corner. In other word, a decrease in $\left(\sigma_{p} / p_{w}\right)_{\max }$ implies that the shock excursion phenomenon is alleviated for the round corner flows.

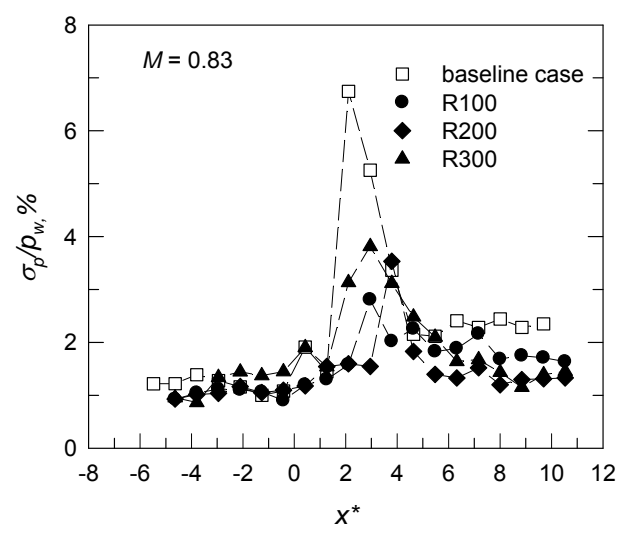

Fig. 4. Distributions of pressure fluctuations, $M=0.83, \eta=15$-deg. 


\subsection{Zero-cross frequency in Round Convex-Corner Flows}

With the interaction of shock wave and boundary layer, oscillatory motion of the separation bubble and shock unsteadiness lead to intense surface pressure fluctuation. At $M=0.83$, the distributions of $f_{c}$ for the round convex-corner flows are shown in Fig. 5 . In the cases of R100 and R200, the level of $f_{c}$ decreases with increasing turning angle as observed in the baseline case. Chung ${ }^{4}$ demonstrated that the peak pressure fluctuations of shock-induced boundary layer separation (SIBLS) in the baseline cases are associated with the induced separation length. As the separation bubble length grows with increasing turning angle, the frequency of separation bubble motion (expansion and contraction) becomes lower. That may explain why the zero-cross frequency has an opposite trend with separation bubble length. As also can be seen in Fig. 5, an increase in the level of pressure fluctuations corresponds to lower zero-cross frequency. Further, in the cases of R300, there is a roughly constant level of surface pressure fluctuations or the variation of pressure fluctuations with the turning angle is less significant. The zero-cross frequency would be independent of turning angle in the cases of R300.

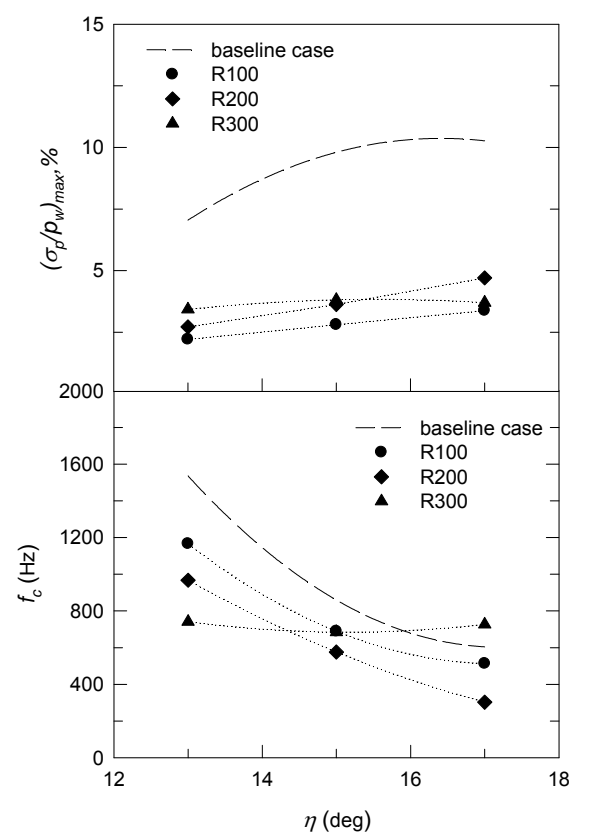

Fig. 5. Zero-cross frequency of round convex-corner flows.

\section{Conclusions}

In this experimental investigation, the flows over compressible round convex- corner at $M=0.64-0.89$ were examined. It was found that the presence of convex surface curvature leads to a decrease in $\left(\sigma_{p} / p_{w}\right)_{\max }$, compared with the test cases of the single convex corner. A lower level of the $\left(\sigma_{p} / p_{w}\right)_{\max }$ corresponds to alleviated shock excursion 
phenomenon. It has been known that the spatial extent of SIBLS is associated with $M_{\text {peak }}$. The maximum wall pressure fluctuations and the zero-cross frequency have an opposite trend. In the cases of R300, the maximum wall pressure fluctuations and the zero-cross frequency are independent of the total turning angle.

\section{Acknowledgments}

This study is under the support of Ministry of Science and Technology (MOST 1032221-E-006-192).

\section{References}

1. A. Bolonkin and G.B. Gilyard, NASA TM-1999-206586 (1999).

2. K.M. Chung, J. Aircraft 37(6), 1079 (2000).

3. K.M. Chung, J. Aircraft 39(6), 1014 (2002).

4. K.M. Chung, Experiments in Fluids 37(6), 917 (2004).

5. K.M. Chung, J. Aircraft 44(6), 1948 (2007).

6. K.M. Chung, J. Aircraft 47(4), 1189 (2010).

7. K.M. Chung, P.H. Chang and K.C. Chang, AIAA J. 50(4), 958 (2012).

8. K.M. Chung, P.H. Chang and K.C. Chang, Science World J. Article ID 940862 (2013).

9. K.M. Chung, P.H. Chang and K.C. Chang, J. Aircraft 50(4), 1011 (2013).

10. D.S. Dolling and M.T. Murphy, AIAA J. 21(12), 1628 (1983).

11. J. Andreopoulos and K.C. Muck, J. Fluid Mechanics 180, 405 (1987).

12. D.S. Dolling and J.C. Narlo, AGARD-CR-428 (1987).

13. T.T. Tran, T. T., PhD thesis, (Aerospace and Mechanical Sciences Department, Princeton University, New Jersey, USA, 1987).

14. M.E. Erengil and D.S. Dolling, D. S., AIAA J. 29(5), 728 (1991).

15. D.S. Dolling and L. Brusniak, AIAA J. 27(6), 734 (1989).

16. S. Pirozzoli and F. Grasso, Physics Fluids 18(6), 065113 (2006).

17. D.S. Dolling, AIAA J. 39(8), 1517 (2001).

18. M. Bernardini, S. Pirozzoli and F. Grasso, J. Fluid Mechanics 671, 288 (2011). 